\title{
Defined Exclusion Criterion
}

National Cancer Institute

\section{Source}

National Cancer Institute. Defined Exclusion Criterion. NCI Thesaurus. Code C93363.

An activity defined at a global library level that identifies a characteristic or requirement intended to be applied to a potential study subject to determine whether they may participate in a study. 